\title{
Russie : Universités et entreprises, le rapprochement de deux mondes
}

Tatiana Kastouéva-Jean

\section{(2) OpenEdition}

1 Journals

Édition électronique

URL : http://journals.openedition.org/ries/2239

DOI : 10.4000/ries.2239

ISSN : 2261-4265

Éditeur

Centre international d'études pédagogiques

Édition imprimée

Date de publication : 1 avril 2012

Pagination : 14-16

ISBN : 978-2-85420-594-7

ISSN : $1254-4590$

Référence électronique

Tatiana Kastouéva-Jean, « Russie : Universités et entreprises, le rapprochement de deux mondes », Revue internationale d'éducation de Sèvres [En ligne], 59 | avril 2012, mis en ligne le 06 février 2015, consulté le 03 mai 2019. URL : http://journals.openedition.org/ries/2239; DOI : 10.4000/ries.2239

Ce document a été généré automatiquement le 3 mai 2019

(c) Tous droits réservés 


\title{
Russie : Universités et entreprises, le rapprochement de deux mondes
}

\author{
Tatiana Kastouéva-Jean
}

1 Pendant la période soviétique, les rapports entre les universités et les entreprises s'organisaient principalement autour de trois axes: stages pendant les études, financement des cursus de certains étudiants par les entreprises qui les embauchaient et, enfin, affectation imposée aux diplômés à la sortie. À quelques exceptions près, l'enseignement (universités), la recherche (instituts de l'Académie des sciences) et le développement/la production (instituts spécialisés/entreprises) étaient cloisonnés.

2 La période de transition et les difficultés financières ont distendu encore plus des liens déjà faibles. Les parties se critiquaient mutuellement. Les universités dénonçaient le manque de volonté des entreprises d'investir dans les formations et récusaient la croyance héritée de l'époque soviétique que le financement des universités était l'affaire de l'État. À leur tour, les employeurs critiquaient une déconnection des universités de l'économie réelle et la baisse de la qualité des diplômés. Les plus grandes entreprises ont préféré investir dans des systèmes de formation internes, appelés "universités corporatives » (Severstal, Rostelecom, Lukoil, etc.). Le système d'enseignement reste encore prisonnier de tout cet héritage : selon les études du Haut Collège d'économie, en 2010 , seulement $40 \%$ des employeurs avaient des liens réguliers avec les universités et la plupart se réduisaient à l'organisation de stages.

3 Cependant, cette situation a commencé à évoluer sous la pression des autorités publiques, qui, depuis 2004-2005, œuvrent au rapprochement entre l'enseignement, la recherche et le développement (R\&D) et l'innovation. Un nouveau modèle d'école supérieure est prôné aujourd'hui, intégrant un cycle complet d'innovation dans ces universités: de la conception de la recherche à sa commercialisation. Le modèle souvent cité est l'université Stanford et la Silicon Valley dont s'inspire, notamment, Skolkovo, "ville innovante " construite à côté de Moscou autour d'une université. La perception du rôle de l'université change en Russie : elle est désormais placée non seulement au cœur de la vie éducative et sociale, mais aussi de la modernisation de son économie. Dans un article daté de fin janvier 2012 dans Vedomosti, le Premier ministre et candidat principal aux prochaines 
élections présidentielles, V. Poutine, estime que «le retour à l'innovation de l'économie russe doit commencer par les universités, qui sont des centres de recherche fondamentale et des pôles de formation des cadres innovants ». Entre 2006 et 2011, une quarantaine d'universités leaders a été sélectionnée sur concours pour réaliser ce modèle. Elles ont reçu des financements substantiels.

Trois objectifs sous-tendent ce changement. D'abord, augmenter la qualité des formations pour les besoins d'une économie innovante et diversifiée dans un contexte de fort déclin démographique, en particulier de la population active. Ensuite, assurer à la Russie une part plus importante sur le marché global des services éducatifs, en améliorant sa place dans les classements internationaux, qui accordent une grande importance aux activités scientifiques des universités. Enfin, les raisons financières n'y sont pas étrangères. En effet, le financement de l'enseignement supérieur par les entreprises représente en Russie 15,6\% des dépenses globales des universités, l'essentiel étant assuré par le budget fédéral $(64,3 \%)$ et les foyers $(20,1 \%)$. Le capital privé est appelé à alléger le fardeau du budget public pour améliorer la situation financière des universités. Ce n'est pas un hasard si tous les généreux appels d'offres publics pour les universités lancés dernièrement exigent l'apport de fonds propres (grâce aux entreprises, aux collectivités territoriales, à la vente des produits de la R\&D universitaire, etc.). Certaines universités ont créé des endowments (fonds de dotation), et une réflexion est en cours sur une meilleure utilisation des réseaux des anciens élèves (alumni).

5 Pour réussir sa nouvelle mission, l'université doit coopérer étroitement avec les entreprises (ainsi qu'avec l'Académie des sciences). La coopération s'organise sur trois plans. Le premier, plus traditionnel, concerne les formations initiales et continues. Il peut s'agir de stages pour étudiants et enseignants, d'achats d'équipements pour des salles de conférences et laboratoires mais aussi de nouvelles formes de coopération. Des professionnels interviennent plus souvent dans les amphithéatres. Dans le but de rapprocher les exigences et de répondre aux mieux aux besoins du recrutement, une Agence nationale des qualifications a été créée, qui réunit universitaires et membres de la Chambre de commerce et d'industrie russe. En échange d'un soutien financier, quelques universités sont même en train de devenir «fournisseurs exclusifs » pour telle ou telle grande société (comme le MIFI, université nucléaire, pour Rosatom). Les deux autres canaux sont la R\&D et l'innovation. Il s'agit de la création commune de laboratoires de recherche, de technoparcs et de start-up. En 2010, pour favoriser ce mouvement, l'État a lancé un appel d'offres pour financer des coopérations entre les universités et les entreprises de pointe: au total, plus de cent projets seront financés à hauteur de 19 milliards de roubles (442 millions d'euros) en 2010-2012. Les entreprises ont désormais leurs représentants aux conseils de tutelle des universités leaders.

6 Le rapprochement entre les universités et les entreprises en Russie s'inscrit dans une tendance globale, observée dans tous les pays développés ou émergents. Leur bien-fondé est aujourd'hui accepté par tous les partenaires en Russie: on y voit un moyen de répondre au mieux aux défis de l'économie moderne en changement permanent et rapide et de la concurrence mondiale. Le processus n'est encore qu'à ses débuts en Russie : les revenus apportés grâce aux contrats avec les entreprises de R\&D au budget d'une université restent infimes; certains accords ne fonctionnent que sur le papier. La Russie est loin de se poser les questions auxquelles se heurtent déjà certains pays développés sur l'adéquation entre le paradigme entrepreneurial et la notion de service public, les besoins sociaux, la liberté académique et les valeurs fondamentales des universités. Aucune 
réflexion n'est pour l'heure engagée pour éviter les écueils sur ce chemin, où la Russie semble s'être bel et bien engagée.

INDEX

Mots-clés : enseignement supérieur, entreprise, université

Palabras claves : enseñanza superior, empresa, universidad

Keywords : higher education, enterprises, universities

Index géographique : Russie

\section{AUTEUR}

\section{TATIANA KASTOUÉVA-JEAN}

Diplômée de l'Université d'État d'Ekaterinbourg, du master franco-russe de relations internationales Sciences-Po/MGIMO (Institut des relations internationales de Moscou) et a également obtenu un DEA de relations internationales à l'université de Marne-la-Vallée. Chercheuse à l'Institut français des relations internationales (IFRI) à Paris, elle travaille sur l'enseignement supérieur, la R\&D, l'innovation et les entreprises en Russie, elle est auteur d'une série de monographies sur les universités russes publiée sur le site de l'Ifri et intervient régulièrement dans les médias français et étrangers sur ces sujets. 